\title{
Awareness of the continuously visible: Information acquisition during preview
}

\author{
MARK W. BECKER \\ Lewis \& Clark College, Portland, Oregon \\ and \\ HAROLD PASHLER \\ University of California, San Diego, La Jolla, California
}

\begin{abstract}
What can we learn about a scene while we stare at it, but before we know what we will be looking for? Three experiments were performed to investigate whether previewing a search array prior to knowing the target allows search to operate more quickly (lower reaction time [RT]), more efficiently (reduced set size slope), and/or by consulting abstract mental representations. Experiment 1 compared RTs for previewed and nonpreviewed arrays, some of which were highly degraded with visual noise. Preview reduced RTs for the noisy displays but did not affect search efficiency. Limited interactions of visual quality and preview suggested that prior exposure allowed the extraction and maintenance of about three abstract identities. If the target was one of those items, the observer responded without searching; if not, the observer searched the remaining items as if there had been no preview. Experiment 2 replicated these findings with less extreme noise. In Experiment 3, subjects previewed 0-6 items of a 12-item display. RTs decreased linearly as the number of previewed items increased from 0 to 3 and then reached a plateau, confirming that the capacity of the representation was about 3 items. Implications for visual awareness are discussed.
\end{abstract}

Our subjective experience of visual awareness would seem to suggest that when we gaze at a complex scene, we simultaneously become aware of a great deal of information about the contents and layout of this scene. It is easy to envision important adaptive advantages to acquiring this kind of detailed information. Nonetheless, a number of writers have argued that this is an illusion (Dennett, 1991, 2002; O'Regan, 1992; but see O'Regan \& Noë, 2001) and that the brain extracts far less detailed information about object identities in a scene than one would naturally suspect. It will be argued below that the kinds of data that have been used to argue for this conclusion have serious and unacknowledged limitations, leaving the question unsettled. The present article develops new techniques with which to ask about what kinds of information are extracted when viewers are allowed to inspect a display of familiar objects prior to searching for something in this display.

\section{Change Detection and Change Blindness}

Beginning in the 1980s, a number of studies examined people's ability to detect changes in displays that remain

This work was supported by National Science Foundation Grant SBR 9729778 and by National Institute of Mental Health Grant R01MH45584. We thank Jeremy Wolfe for suggestions that led to Experiment 3 and Sara Vera for her assistance in data collection and analysis. Correspondence concerning this article should be addressed to M. W. Becker, Department of Psychology, Lewis \& Clark College, 0615 SW Palatine Hill Rd., Portland, OR 97219 (e-mail: mbecker@lclark.edu). present for some period of time, disappear, and then reappear (sometimes with alterations). When the disappearance is extremely brief (under about $20 \mathrm{msec}$ ), people are usually very good at detecting such changes, apparently because the changes are detected by relatively peripheral visual mechanisms (Phillips \& Singer, 1974; Stelmach, Bourassa, \& Di Lollo, 1984). When the disappearance is long enough to generate a perception of flicker, however, change detection is quite poor, whether the display consists of an array of letters (Pashler, 1988) or a scene (Levin \& Simons, 1997; O’Regan, Deubel, Clark, \& Rensink, 2000; O’Regan, Rensink, \& Clark, 1999; Rensink, O’Regan, \& Clark, 1997; Simons \& Levin, 1997). This surprisingly poor detection of change has sometimes been termed change blindness, although this term appears to overstate the phenomenon, given that observers sometimes retain several objects, probably the full complement of what they can store in visual short-term memory (VSTM; Pashler, 1988), and successfully detect changes that occur to those items. One might suppose that relatively poor change detection reflects the fact that observers have not expended the effort needed to identify those objects whose identities are subsequently changed, but studies that have required observers to demonstrate that they have exhaustively identified an entire array of characters nonetheless have shown that when the display subsequently flickers on and off, the usual deficit in change detection is present (Becker \& Pashler, 2002).

These results from change detection studies are certainly consistent with the idea that people are aware only 
of a tiny subset of items at any instant of time and that the ability to maintain these representations over time and across saccades is fairly limited. However, as has been noted elsewhere (Becker, Pashler, \& Anstis, 2000; Hollingworth, 2003; Simons, 2000), the method used in these change detection experiments is not ideally suited for investigating the quantity of information a person has about a scene while that scene is in view. The task requires an observer to compare a scene that appeared at one time with a scene that appears at another time. Consequently, it requires the observer not only to represent the to-bechanged item while the scene is present, but also to store this representation and to utilize some mechanism capable of comparing this representation with the information presented in the second display. Thus, a failure to detect a change may reflect the inability of the visual system to simultaneously represent and compare two complete scenes, rather than a failure to represent objects while they are present in the environment.

A number of recent findings provide some encouragement for the suspicion that poor change detection might underestimate the extent of the information represented while scenes are viewed. For example, Hollingworth (2003) found that a postdisplay cue at the location of a change improved change detection accuracy. Presumably the postdisplay cue allowed the observer to focus his/her comparison on one location. A number of similar findings have been taken to suggest that visual representation may be more detailed than traditional change detection paradigms suggest (Hollingworth \& Henderson, 2002; Hollingworth, Williams, \& Henderson, 2001; Simons, Chabris, Schnur, \& Levin, 2002). In addition, implicit measures of change blindness suggest that explicit change detection may underestimate the amount of information that is represented (Fernandez-Duque \& Thornton, 2002; Thornton \& Fernandez-Duque, 2000; but see Mitroff, Simons, \& Franconeri, 2002).

In an effort to account for these findings, Hollingworth and colleagues (Hollingworth, 2003, 2004; Hollingworth \& Henderson, 2002; Hollingworth et al., 2001) proposed that visual representations are made up of both a limited capacity VSTM component and a fairly high capacity visual long-term memory (VLTM) component. In their model, the VLTM store allows people to build up a much more complex representation of the visual world than traditional change blindness experiments suggest.

Clearly, there is no general consensus from the change detection literature concerning the amount of information available while a scene is viewed. In addition, the method may not be able to definitively answer this question.

\section{Effects of Preview}

Another, rather different approach to determining what kind of information is available when a display is viewed is to look at the consequences of previewing a display for subsequent judgments performed on the same display. Pashler (1984) had subjects view displays of eight letters, some of which were visually degraded (by reducing either the contrast or the discriminability of the stimuli). At a variable point in time, relative to the array, a bar marker cue was presented, singling out one character; the subject's task was to make a speeded response dependent upon the identity of this character. In some cases, the display was made available for a brief preview before the cue appeared, and in other cases, the cue appeared slightly before the display.

If observers construct an abstract representation of the contents of the display while they preview the display, the preview should eliminate the effect of visual quality on the reaction times (RTs) for the speeded response to the cued character. However, this is not what the results showed. The effects of degradation on RTs were still present even after preview; in fact, the effects of reduced target discriminability were sometimes enlarged, as compared with the no-preview conditions. The results imply that even when observers are given the opportunity to preview a display and have an incentive to process as many items as possible, the probed item nonetheless is identified after the probe has arrived. This finding held true even when unpredictable catch trials were introduced in which the display was masked immediately after preview, in order to give subjects every incentive to process the display as extensively as possible during the preview. Mewhort, Johns, and Coble (1991) confirmed this basic result (persistence of the degradation effect after preview), using a different manipulation of degradation: dots superimposed on top of characters.

These results are certainly consistent with the suggestion that people cannot build a rich representation of the identities and layouts of a multielement display containing eight familiar objects. However, other interpretations are possible. For example, observers might construct such a representation but be unable to use location as a cue for retrieving identity information from it. This might occur if the information represented was equivalent in content to a list of the items present in the display. This list could even contain directional pointers linking the items to their respective locations, but if there were no pointers in the opposite direction, cuing a location might not access the identity of the object at that location (Pashler \& Badgio, 1985).

\section{Repeated Search Effects}

A third way to determine what information observers can extract from a scene involves requiring people to search a single display repeatedly. Wolfe, Klempen, and Dahlen (2000) modified the standard visual search procedure so that the same display was searched on multiple consecutive trials, with the search target changing from trial to trial. In their experiments, an array of items appeared on the screen, with the target presented in the center of the array as either a written description or a picture. After the observer responded, a new target specification appeared, and the subject had to search the same display 
for the new target. In this method, a single scene remained present throughout the task, and the task did not require either retention (as in the change detection task) or retrieval of identities on the basis of locations (as in the previewed partial-report design of Pashler, 1984).

One might suppose that the first search of the array would be slow and inefficient but that, after repeated searches of the same array, the observer would become familiar with the items of the display and the search process would become more efficient or even be dispensed with altogether. To the contrary, Wolfe et al. (2000) found that the slope of the search function (milliseconds per item search rate) remained the same as the display was repeatedly searched. However, there was a reduction in overall RT from the first to the second search, with overall RTs remaining constant thereafter. The authors concluded that observers did not acquire knowledge of the scene that could be used to bypass the need to search, suggesting that the postattentive representations were quite impoverished.

Why would an observer continue to search the same display over and over? One possibility is that the visual system never constructs a detailed representation of the visual scene. A second possibility is that the visual representations constructed during a search task may be task specific. For example, if an observer searches for an $A$, the mental representation may treat any letters other than $A$ as a token of an undifferentiated category, not- $A$. Thus, the representation that is generated by the search may assert the presence of an $A$ surrounded by not- $A$ s. If so, when the subject must then search the display for a target $C$, there will be no benefit.

A third possibility is that observers may be able to create a rich representation of identities and their locations and would even have been able to use it, except for a special feature of Wolfe et al.'s (2000) experiment: the demand to acquire the target instruction through the visual modality. One would expect that this would require a redeployment of visual attention, perhaps narrowing attention onto the target instruction, which might interrupt the processing of the rest of the display and purge any putative rich representation of the array.

A final possibility is that the visual search in this task is so rapid and easy that even if observers have a potentially usable internal representation of the array, they may fail to consult it, simply because doing so will provide little benefit (while perhaps imposing some unknown costs). To shed light on this possibility, Wolfe et al. (2000) had subjects memorize a display and then removed the display and had the subjects perform the target present/absent decision from memory. They compared these subjects' performance with that of subjects who searched the same three-item visual display for 350 trials. Even though the latter subjects clearly had memorized the three letters by the 350th trial, they never responded as quickly as the memory search subjects. These subjects should have been able to respond more quickly by switching to a memory search; instead, they continued to perform a visual search, probably because a memory search is more effortful than a simple visual search. A similar explanation might account for the failure to find a speedup for subsequent trials in a repeated search task. Subjects might be able to compare the target item with an internal visual representation of the display; but when the subject is given simple visual displays, the benefit of doing so may not be worth the extra effort. If so, it would be a mistake to see the results as arguing against the existence of a rich representation of identities and their layouts.

\section{Current Approach}

The goal of the present study was to shed new light on the question of what kind of information observers can represent when they view a scene composed of a number of familiar objects, with the number exceeding the capacity of visual working memory (typically, three or four). We asked how preview of an array would interact with both the number of objects in the array (as in Wolfe et al., 2000) and the visual quality of these items (as in Pashler, 1984). Unlike Pashler (1984), however, we used a search task that required only knowledge of what was present in the display, rather than retrieval of an identity on the basis of its location. Unlike Wolfe et al. (2000), the target letter was communicated to the subject aurally, through computer speakers. The purpose of the aural presentation was to avoid forcing the observer to shift visual attention between the time when the subject was given the opportunity to preview the display and the time when the information present was assessed.

The subjects saw a display composed of 3, 6, or 12 letters. On half the trials, the letters were visually degraded, much as in Mewhort et al. (1991). The subjects' task was to determine whether a target letter (which varied from trial to trial) was present in the display.

The most critical manipulation was the relative timing of the two types of information (visual array and aural cue). In some trials, the subjects had the opportunity to preview the display for $2 \mathrm{sec}$ before hearing the identity of the target. In other trials, the target was specified $2 \mathrm{sec}$ before the display was presented. If an observer can construct a mental representation of all 12 identities present in the display (whether or not this is tied to the location or addressable by a location cue), he/she should be able to determine whether the specified target is present, without having to consult the low-level visual representation of the display. Thus, the effect of degrading the characters should disappear. If people are unable to construct such a representation (even for a single item), preview should not interact with the effects of either display set size or visual quality: They should basically have to start from scratch, and both variables should impose their full costs. A third, fairly obvious possibility, suggested by the nonzero levels of performance seen in change detection (Pashler, 1988) and by the basic findings of the classic visual memory studies (Sperling, 1960), is that subjects might be able to construct an abstract representation of just a few items 
and retain this information in VSTM. In that case, when the aurally presented item is represented within the contents of VSTM, responses could be made without waiting for a search of the display. When the target was present elsewhere in the display (or not present at all), the display would have to be searched.

\section{GENERAL METHOD}

\section{Subjects}

The subjects were undergraduate psychology students with normal or corrected-to-normal vision, participating for course credit.

\begin{abstract}
Apparatus
All the experiments were programmed in Macromedia Director and run on a Power Macintosh 5400 with a 15-in. display running at $66 \mathrm{~Hz}$.

\section{Stimuli}

Visual arrays consisted of 3,6, or 12 letters equally spaced around an imaginary clock face with a diameter of $8^{\circ}$ of visual angle and a small black fixation cross at the center, which the subjects were instructed to fixate until they heard the identity of the search target. Letters were black 48-point Times New Roman font letters (subtending $\sim 1.2^{\circ} \times 1.5^{\circ}$ at $8^{\circ}$ eccentricity) presented on a white background. To create a given array, letters were randomly selected without replacement.

Targets were presented aurally at about $90 \mathrm{~dB}$ through stereo speakers placed on both sides of the monitor. The wave files for the letters were created by digitally recording the spoken name of each letter. Each file was then compressed or expanded, using Sound Edit 16 , until each of the recordings was $450 \mathrm{msec}$ long.
\end{abstract}

\section{EXPERIMENT 1}

\section{Method}

The design was a $3 \times 3 \times 2$ within-subjects design with factors of timing, set size, and visual quality. The timing variable consisted of three different stimulus onset asynchronies (SOAs) between the visual search array and the aurally presented target (see Figure 1) and was varied across blocks of trials. In target-first blocks, the target was aurally presented $2 \mathrm{sec}$ before the onset of the visual array. In simultaneous blocks, the target was presented at the same time as the onset of the search array. In visual-first blocks, the visual search array was presented $2 \mathrm{sec}$ prior to the presentation of the target.

Each block consisted of 84 trials made up of 14 target-present trials and 14 no-target catch trials at each set size (3, 6, and 12). Half (7) of the target-present trials in the block had displays in which the visual letters were clear black letters on a white background; in the other half, all of the letters were degraded by embedding them in random visual noise (see Figure 2). Similarly, half of the notarget catch trials had displays with noise, and half had clear displays. Thus, within a block, there were 7 trials within each set size $X$ present/absent $X$ visual quality cell of the experiment. The visual noise consisted of a rectangular patch $\left(\sim 2.5^{\circ} \times 2.2^{\circ}\right)$, which was superimposed on top of each letter in the display. The noise patches were $60 \times 60$ pixels, and each pixel had a $60 \%$ chance of black.

In all blocks, the measurement of RTs started as soon as the presentation of both pieces of information (display and spoken name of target) had begun. After the subject's response, the screen was cleared for $2 \mathrm{sec}$ before the next trial began.

At the end of each block, a screen announced the next block's timing condition, and the subject hit any key when he or she was ready to begin. Each of the three types of block occurred twice, and the order of blocks was randomized. Each subject $(N=30)$ participated

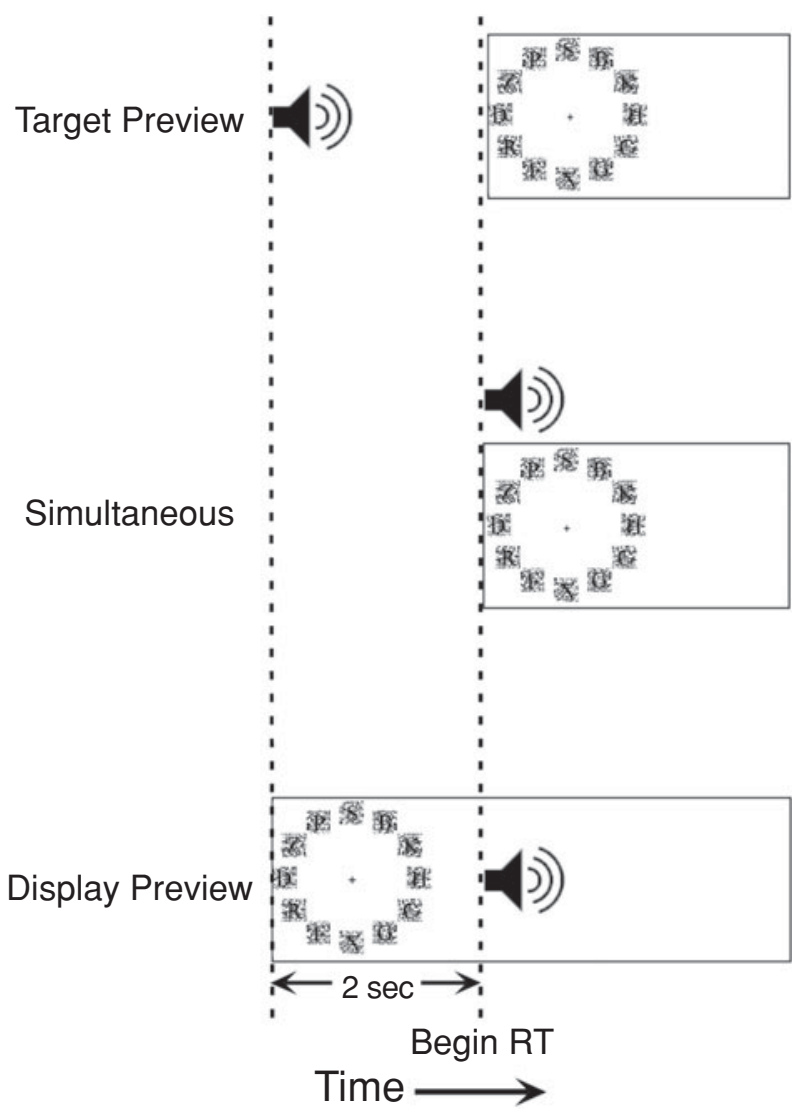

Figure 1. The search target for each array was presented by the speaker. The onset of the visual array and the delivery of the target was varied across three timing conditions. Once a display had appeared, it remained on the screen until the observer's response. The reaction time (RT) timing began once the display was visible and the target was known. The schematic depicts 12 items embedded in visual noise; half the trials did not have visual noise, and there were additional set sizes of 3 and 6 items.

in a total of six blocks (for a total of 504 trials). A 5-min break occurred between the third and the fourth blocks of trials.

\section{Results}

RT data. Mean RTs and standard deviations for each condition are presented in Table 1. Figure 3, panel A shows the mean RTs for hits. There are a number of findings worth noting in the overall RT data. First, adding visual noise to the displays slowed responses considerably. This is apparent from the figure and is verified by the significant main effect of visual quality $[F(1,29)=$ $601.07, p<.01]$.

Second, previewing the display had differential effects depending on whether or not the display contained noise, producing a significant visual quality $\times \mathrm{SOA}$ interaction $[F(2,58)=106.43, p<.01]$. Preview failed to result in faster searches for clear stimuli, suggesting that the observers failed to acquire and use an internal representation with clear stimuli. However, the observers responded more quickly when allowed to preview a search array that 


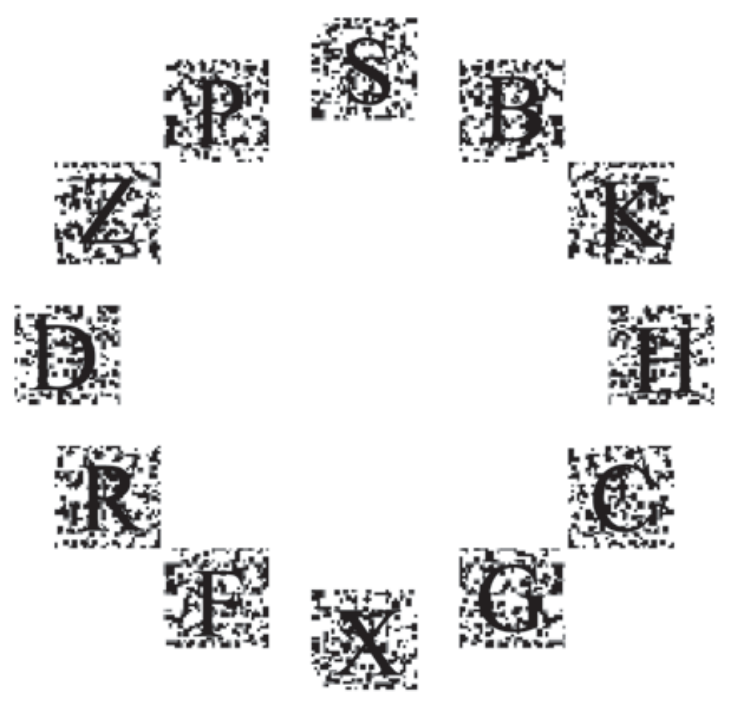

Figure 2. An example of the stimuli for set size 12 with black visual noise. Experiment 2 contained the same amount of noise, but the noise was a mid-gray, rather than black.

contained visual noise, suggesting that the observers acquired information during the preview that allowed the observers to respond to the search task more quickly.

This set of findings is consistent with the hypothesis that observers represent some information during preview but, given clear stimuli, do not base their responses on the information they have acquired and, instead, perform a visual search based on current visual input from the display. Thus, the existence of the representation is evident only when the visual search task is made difficult by embedding the stimuli in visual noise.

Third, previewing a noisy display significantly reduced search times, relative to nonpreviewed noisy displays. Preview completely eliminated the effect of visual noise for 3 -item displays, resulting in RTs similar to those for clear displays. For displays containing 6 or 12 noisy items, preview produced intermediate RTs; they were shorter than those for nonpreviewed noisy searches, but not as short as those for searches of clear items. If preview allowed an ob- server to represent the identity of all the items, the person should have been able to respond at least as quickly, if not more quickly, as when responding to clear items without preview. Thus, these findings suggest that observers are able to represent the identity of at least 3 items but are not able to represent as many as 6 items.

In summary, the RT data suggest the existence of an internal representation that contains information that can be used to perform visual search-information that will be used when the search task is made sufficiently difficult. However, preview completely eliminates the effect of noise only for small set sizes, suggesting that the capacity of this postcategorical representation is severely limited.

Slope data. The slope of the set size search function (milliseconds/item) is often taken as a measure of search efficiency. ${ }^{1}$ Examining slopes may suggest a different interpretation of the benefit of preview.

From Figure 3, it is apparent that the slopes of the set size functions for noisy displays (slope $=51$ for simultaneous and 59 for target-first SOAs) are steeper than the slopes for clear displays (slope $=39$ for simultaneous and 36 for target-first SOAs). These steeper slopes indicate a less efficient search. The main question of interest is whether previewing a noisy display results in a more efficient search. The answer is that after previewing a noisy array, the observer searched the displays at the same inefficient rate (slope $=64$ ) as when not given a preview. This finding suggests that observers represent little information during preview; they fail to perform any type of perceptual filtering or cleaning up of the display that would allow search to proceed from item to item more efficiently.

Figure 3, panel B presents the data for correct rejections on target-absent trials. The pattern of results is identical to the hit data. Applying the logic above to the correct rejection data, the speeding up in overall RT due to preview suggests that a limited capacity representation is created during preview that will be used if the search task is made sufficiently difficult by adding visual noise. The slope data, however, suggest that the representation created during preview does not result in a more efficient millisecond/item search rate.

Table 1

Means and Standard Deviations for Experiment 1

\begin{tabular}{|c|c|c|c|c|c|c|c|c|}
\hline & \multirow[b]{2}{*}{ Display Type } & \multirow[b]{2}{*}{ Preview Condition } & \multicolumn{2}{|c|}{ Set Size 3} & \multicolumn{2}{|c|}{ Set Size 6} & \multicolumn{2}{|c|}{ Set Size 12} \\
\hline & & & $M$ & $S D$ & $M$ & $S D$ & $M$ & $S D$ \\
\hline \multirow[t]{4}{*}{ Hits } & \multirow[t]{2}{*}{ Clear } & Display & 731.33 & 149.15 & 828.69 & 132.74 & $1,026.86$ & 194.53 \\
\hline & & Simultaneous & 733.18 & 93.57 & 868.16 & 114.71 & $1,119.64$ & 189.90 \\
\hline & \multirow[t]{2}{*}{ Noisy } & Display & 759.96 & 158.33 & $1,017.29$ & 227.16 & $1,346.37$ & 293.99 \\
\hline & & Target & $1,157.44$ & 165.05 & $1,373.96$ & 210.03 & $1,695.40$ & 342.38 \\
\hline \multirow{5}{*}{ Correct rejections } & \multirow{2}{*}{ Clear } & Target & 792.29 & 150.03 & 937.90 & 207.56 & $1,529.27$ & 398.20 \\
\hline & & Simultaneous & 857.86 & 168.58 & $1,008.41$ & 202.25 & $1,634.03$ & 401.82 \\
\hline & \multirow[t]{3}{*}{ Noisy } & Display & 863.57 & 181.64 & $1,284.02$ & 321.92 & $2,116.36$ & 546.50 \\
\hline & & Target & $1,249.86$ & 190.49 & $1,822.09$ & 313.30 & $2,525.19$ & 522.65 \\
\hline & & Simultaneous & $1,226.58$ & 186.51 & $1,831.96$ & 311.58 & $2,543.34$ & 602.89 \\
\hline
\end{tabular}



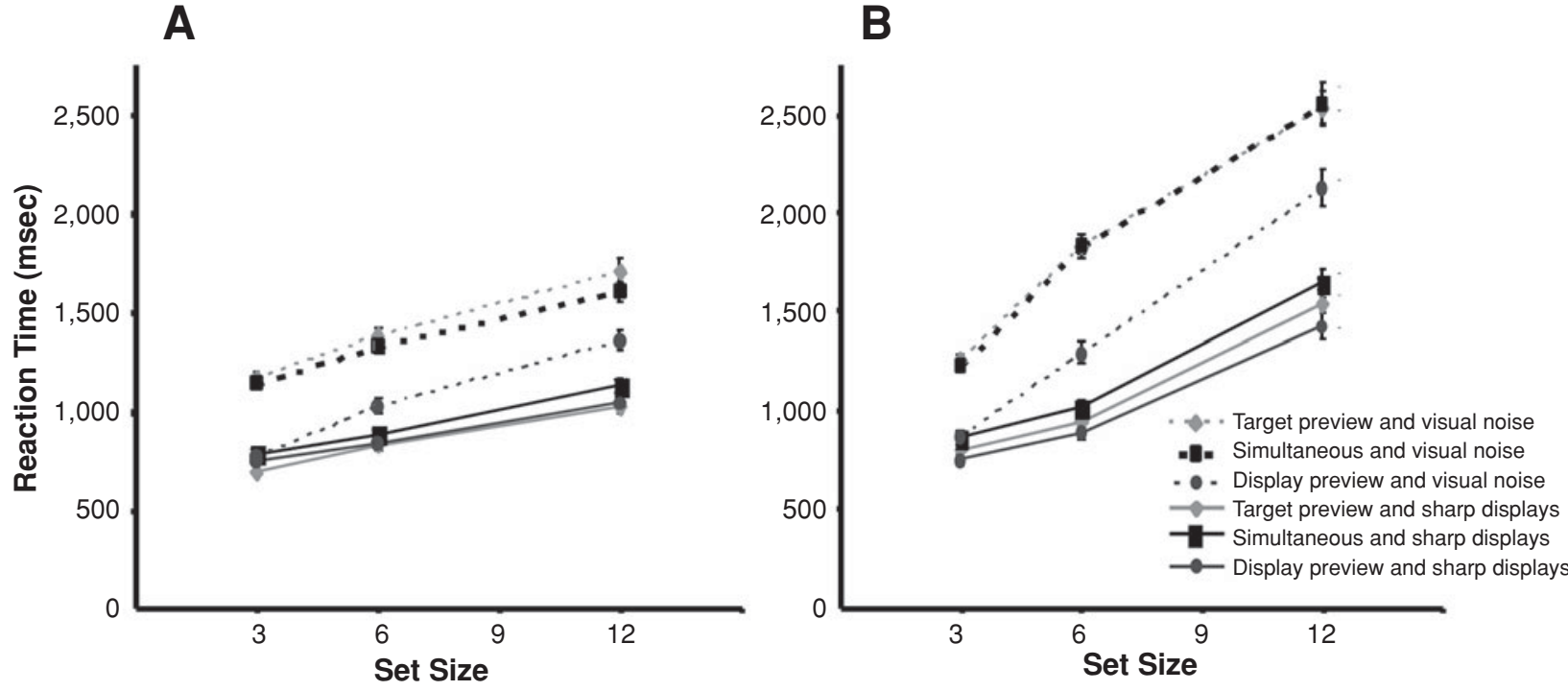

Figure 3. Reaction time data for hit trials (panel A) and correct rejection trials (panel B) in Experiment 1. Solid lines indicate clear visual displays. Broken lines indicate arrays embedded in visual noise. Dark gray circles indicate trials in which the search array was previewed for 2 sec prior to the announcement of the identity of the search target. Trials in which the visual array was not previewed are indicated by the black squares (simultaneous onset of the array and search target) and gray diamonds (the identity of the search target was revealed 2 sec before the search array appeared).

In summary, the data show that previewing a noisy array leads to a reduction in overall RT or a change in the $y$-intercept of the set size search function. This finding suggests that some information is being extracted during preview that is allowing the observer to complete the search task more quickly. However, the slope data suggest that the observer searches a noisy array at the same (relatively inefficient) rate regardless of whether he/she has previewed the display. The question of how to interpret a change in $y$-intercept without a change in search slope is a difficult one. Wolfe et al. (2000) also had difficulty interpreting this pattern in their results. They proposed that the change in $y$-intercept was an artifact caused by the simultaneous presentation of a target and a search array and based their interpretations exclusively on the slope data. However, we will offer a model below that accounts for both the slope and the intercept data. The model assumes that an observer is able to represent the identity of about three items from the display but retains no information about the remaining items.

\section{Discussion}

There seems to be little doubt that during preview, people were unable to acquire an internal representation of the identities of all the items when 6 or 12 characters appeared on the screen. If they had been able to do so, degrading the visual image should have had no effect on RTs to previewed displays. Conversely, degrading the image had no effect when the observers were allowed to preview a display with only three items, suggesting that people can internally represent at least three items. But how does this ability to represent a limited number of items affect mean
RT when a degraded display contains more items than an observer can represent?

We suggest a possible interpretation. During preview, subjects can identify and internally represent a limited number of items (at least three in the present situation). If the target letter ends up being one of the letters that the subject has represented, the subject is able to answer rapidly without searching the physical stimulus on the screen. However, if the target is not one of the attended letters, the subject must return to the display and search the remaining items. During this search of the remaining items, there is no benefit of having previewed the display, thus generating the steep slope of the search function for the visually previewed displays with random noise.

The question remains, when the target letter is not one of the letters that the observer mentally represents, does the observer have to search all the locations in the display, or are the locations of represented items eliminated from this search? The correct rejection data show that subjects benefit from a visual preview of letters embedded in visual noise. This advantage implies that the locations of represented items are eliminated from the subsequent search.

If this interpretation is correct, it makes strong predictions about the distributions of RTs for conditions in which an observer is allowed to preview a visual array with noise. These distributions should represent a mixture of two types of trials. On some trials, the target should be one of the items the subject represented during preview, and thus the RTs should be relatively short. On the remaining trials, the target would not be one of the represented letters, and thus the RTs should be relatively long. More specifically, these RTs should reflect the distribution of 
RTs for trials in which the observer searches a noisy display with a set size equal to the number of items in the display minus the number of items represented.

This analysis can be expressed mathematically in the following way:

$$
\begin{aligned}
\mathrm{RT}(X \text { noisy items })= & \frac{C}{X}[\mathrm{RT}(\text { memory })]+\frac{X-C}{X} \\
& \cdot\{\operatorname{RT}[(X-C) \text { noisy items }]\},
\end{aligned}
$$

where $C$ is a constant denoting how many items an observer can represent in memory, $C / X \cdot[\mathrm{RT}$ (memory) $]$ is the proportion of trials on which the subject represents the search target during preview, and $(X-C) / X \cdot\{\mathrm{RT}[(X-C)$ noisy items] $\}$ is the proportion of trials on which the subject searches the nonrepresented items of the display.

Applying the same logic to correct rejection trials produces a slightly different formula. In correct rejections, the target item is never in the display; therefore, if the set size is less than the number of items the subjects can represent (the constant $C$ ), the subjects respond quickly on the basis of this representation. However, if the set size is greater than $C$, the number of trials based on the internal representation becomes zero, and the formula is reduced to

$$
\mathrm{RT}(X \text { noisy items })=\mathrm{RT}[(X-C) \text { noisy items }] .
$$

If we assume that a previewed display with a set size of three produces RTs similar to those for clear displays with a set size of three, because the subject is able to represent at least three items, we can substitute the mean RT for three clear items for the RT from memory term in the equation. Using the mean regression line for the noisy stimulus, we can predict the RTs that should be observed for hits and correct rejections, using the above formulas. Setting $C$ (the number of items represented) equal to 3.13 minimizes the errors of the predictions and produces the predicted values shown in Figure 4. These predicted values are very similar to the observed values.

We conclude that previewing a display that contains visual noise may decrease the overall RT for two reasons. First, it allows subjects to make a rapid response without searching on the portion of trials in which the target is one of the items that the observer has internally represented. Second, even when the target is not internally represented during the preview, RTs are reduced because the subsequent search need not reexamine locations that have been stored in memory. Although both of these effects lower the overall RT, neither of them affects the overall search efficiency, resulting in no change of slope but a change in the $y$-intercept. This explanation suggests that the $y$ intercept effect should not be ignored. Instead, a change in $y$-intercept in the absence of a change in slope can indicate that information, albeit limited, is being extracted from the scene during preview.

Using such severe noise produced an interaction that allowed us to determine that observers still search through the stimuli on the screen after previewing a noisy display, which allowed us to test our model. However, in order to produce this interaction, we used an atypical amount of visual degradation. To test the generalizability of this model, we sought to apply it to another set of data derived from a visual quality manipulation that is more typical of visual quality manipulations in the literature. Several researchers who have examined visual quality effects in search have found these effects to be additive with display set size (Egeth \& Dagenbach, 1991; Logan, 1978; Mewhort et al., 1991; Pashler \& Badgio, 1985). These authors generally concluded that degrading the visual quality of a display affects only the sensory encoding stage of the search task and that encoding can be done in parallel across the visual field.

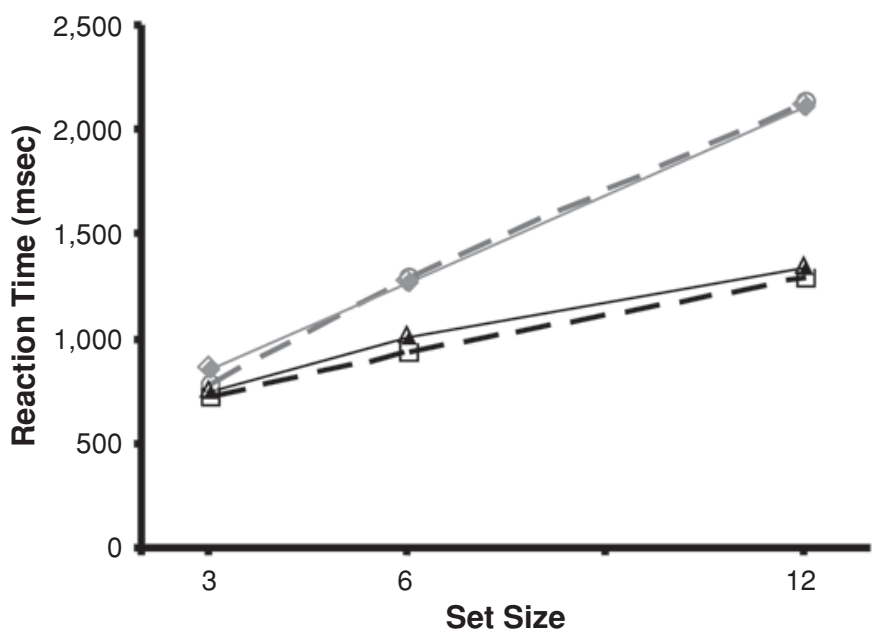

Figure 4. The predictions of the regression model (broken lines) compared with the observed values (solid lines) for the condition in which an array containing gray random visual noise was previewed. The gray lines indicate correct rejection data, and the black lines indicate hit data. 
The severity of the visual degradation in Experiment 1 was much greater than the degradation used in these earlier studies. In Experiment 1, the average slowing caused by the visual degradation was approximately $500 \mathrm{msec}$ for hits and over $700 \mathrm{msec}$ for correct rejections. The quality manipulations in the works cited above generally resulted in delays of less than $100 \mathrm{msec}$. There is, however, one study that comes close to the level of degradation we use in Experiment 1. Johnsen and Briggs (1973) obtained an effect of about $345 \mathrm{msec}$, and indeed, they found a set size $\times$ visual quality interaction in one of their conditions.

Perhaps, then, the degradation used in Experiment 1 was so severe that it resulted in an unusually difficult visual search task, one in which each item was so severely degraded that attention had to be allocated to the item in order to complete the encoding phase (transforming the encoding process, rather than merely slowing it, as in a traditional additive factors experiment; cf. Sternberg, 1969). If so, the conclusions based on Experiment 1, although still informative about the total information that one can extract from a scene, given preview, may differ in important ways from more typical visual search experiments.

In order to test this possibility, we performed a second experiment that was identical to Experiment 1, but with a less severe degradation of the visual stimulus in the visual noise condition.

\section{EXPERIMENT 2}

\section{Method}

The method was identical to that in Experiment 1, except that the black visual noise was now replaced by a gray visual noise. Since the letters were black, this manipulation seemed likely to allow the observers to distinguish the letter from the noise with much less difficulty.

\section{Results and Discussion}

The RTs for hits and correct rejections are plotted in Figure 5 (and see Table 2). The pattern of data is very similar to that in Experiment 1. A repeated measures ANOVA produced all of the same significant main effects and interactions as those in Experiment 1, with one notable difference. The less obtrusive gray noise in this experiment resulted in a roughly additive effect with set size. A repeated measures ANOVA on the data for which the preview condition was excluded showed no set size $X$ visual stimulus quality interaction $[F(2,40)<1]$, suggesting that with this level of stimulus degradation, our results are in closer agreement with previous findings that visual quality manipulations produce additive effects.

However, it is worth pointing out that the visual quality $\times$ set size interaction was significant when the visual preview data were included in the analysis. Even if the reduction of noise was processed in parallel, the model we presented to explain our results might produce a significant interaction when the preview condition is added into the analysis. The relative proportion of the two types of RTs - those when the target is one of the represented items and those when it is not - changes with set size and, thus, might produce a significant interaction. Thus, we believe that the stages slowed by noise in this experiment were largely carried out in parallel (cf. Pashler \& Badgio, 1985 ) and that this experiment is a replication of Experiment 1 with stimuli that more closely represent the types of stimuli used in typical visual quality manipulations.
A

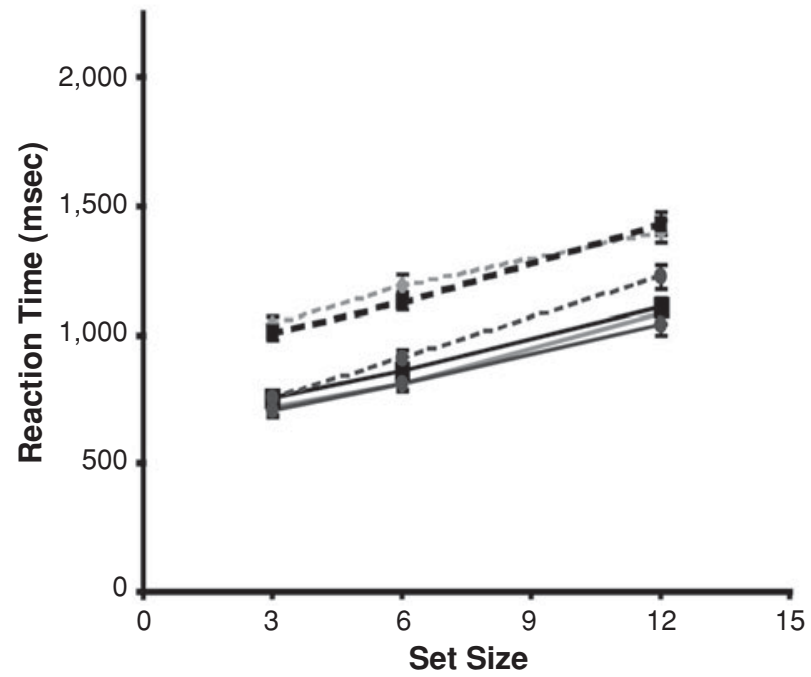

B

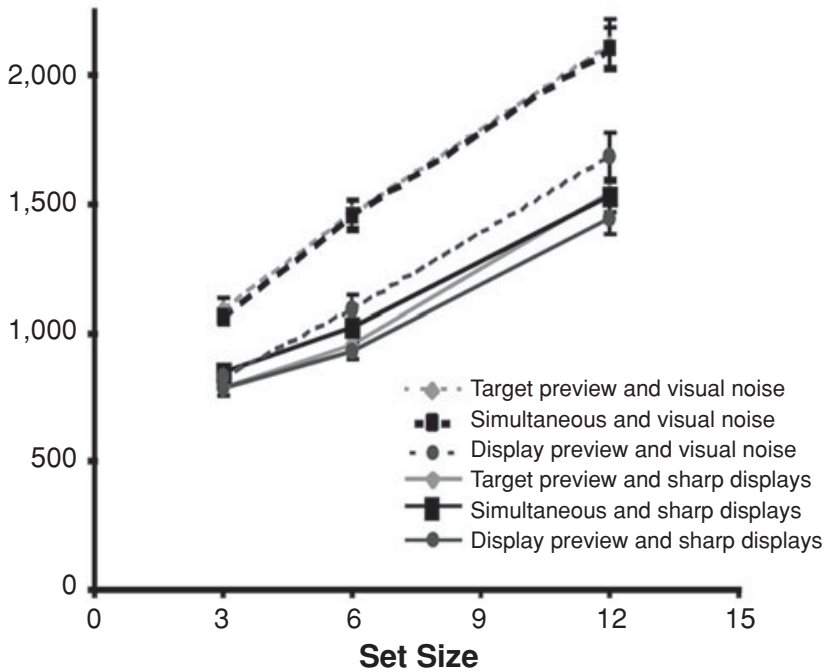

Figure 5. Panel A plots the hit data, and panel B plots the correct rejection data in Experiment 2. Solid lines indicate clear visual displays. Broken lines indicate arrays embedded in visual noise. Dark gray circles indicate trials in which the search array was previewed for 2 sec prior to the announcement of the identity of the search target. Trials in which the visual array was not previewed are indicated by the black squares (simultaneous onset of the array and search target) and gray diamonds (the identity of the search target was revealed 2 sec before the search array appeared). 
Table 2

Means and Standard Deviations for Experiment 2

\begin{tabular}{|c|c|c|c|c|c|c|c|c|}
\hline & \multirow[b]{2}{*}{ Display Type } & \multirow[b]{2}{*}{ Preview Condition } & \multicolumn{2}{|c|}{ Set Size 3} & \multicolumn{2}{|c|}{ Set Size 6} & \multicolumn{2}{|c|}{ Set Size 12} \\
\hline & & & $M$ & $S D$ & $M$ & $S D$ & $M$ & $S D$ \\
\hline \multirow[t]{6}{*}{ Hits } & \multirow{3}{*}{ Clear } & Display & 703.99 & 114.25 & 813.82 & 123.25 & $1,040.45$ & 184.67 \\
\hline & & Target & 717.11 & 129.28 & 814.54 & 154.20 & $1,085.01$ & 191.19 \\
\hline & & Simultaneous & 757.14 & 82.82 & 862.64 & 105.24 & $1,110.51$ & 145.54 \\
\hline & \multirow[t]{3}{*}{ Noisy } & Display & 754.49 & 126.62 & 913.06 & 141.87 & $1,226.09$ & 217.62 \\
\hline & & Target & $1,043.64$ & 145.27 & $1,193.06$ & 196.56 & $1,400.61$ & 205.56 \\
\hline & & Simultaneous & $1,009.01$ & 127.10 & $1,129.69$ & 149.32 & $1,433.13$ & 201.41 \\
\hline \multirow{6}{*}{ Correct rejections } & \multirow{3}{*}{ Clear } & Display & 789.11 & 138.26 & 930.15 & 147.56 & $1,444.26$ & 292.18 \\
\hline & & Target & 786.55 & 125.81 & 955.88 & 223.12 & $1,544.49$ & 304.15 \\
\hline & & Simultaneous & 848.37 & 99.30 & $1,021.28$ & 160.30 & $1,529.72$ & 270.96 \\
\hline & \multirow[t]{3}{*}{ Noisy } & Display & 833.57 & 137.77 & $1,091.51$ & 248.93 & $1,688.22$ & 402.47 \\
\hline & & Target & $1,093.78$ & 178.31 & $1,464.41$ & 254.73 & $2,118.78$ & 453.22 \\
\hline & & Simultaneous & $1,063.56$ & 120.44 & $1,454.04$ & 281.00 & $2,110.33$ & 365.82 \\
\hline
\end{tabular}

The main goal of this replication was to determine whether the mixture model derived to explain the data from Experiment 1 could also explain data for a more typical visual noise manipulation. To make this comparison, we used the same two formulae but used the regression line for searching for letters embedded in gray noise, rather than in black noise, to predict the time taken to search noisy items. Setting the capacity of the mental representation to 3.18 items minimized the errors between the predictions and the observed data and produced a fairly good fit (see Figure 6). The similarity of the estimate to that of 3.13 in Experiment 1 is quite encouraging.

\section{EXPERIMENT 3}

The results from Experiments 1 and 2 suggest that the representation formed while a display is previewed is subject to a capacity limitation, maintaining up to about three items (in the present situation). A third experiment directly assessed the capacity of the representation formed during preview by varying the number of items available during preview. If the representation formed during preview can hold only about three items, we would expect search times to decline as the number of items previewed increased from one to three items. With three previewed items, the representation capacity should be full; thus, adding additional items to the preview should be of no additional benefit.

\section{Method}

Displays were identical to the 12-item displays in Experiment 1 (12 letters embedded in black visual noise, displayed around an imaginary clock face). There were 24 trials ( 12 containing a search target and 12 catch trials) in each of seven preview conditions, for a total of 168 trials. Each trial began with a 2 -sec preview of between 0 and 6 of the 12 items and the noise in which they were embedded. ${ }^{2}$ Items in the preview were adjacent to one another and remained in the same location when the rest of the display appeared. For example, for the 3-item preview condition, the previewed items might be located at clock positions 10 through 12; after $2 \mathrm{sec}$, the rest of the items appeared at positions 1 through 9 . The target of the

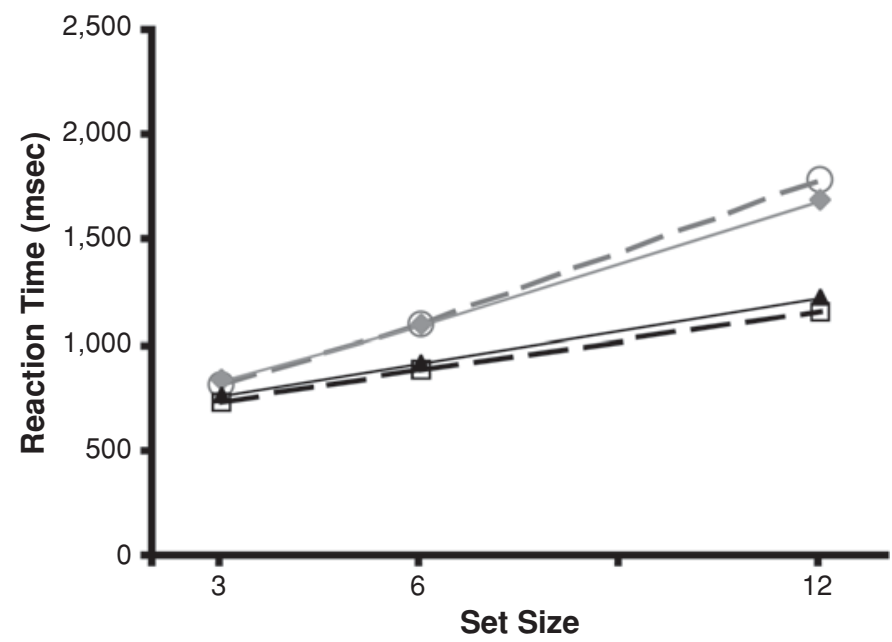

Figure 6. The predictions of the model (broken lines) compared with the observed values (solid lines) for the condition in which an array containing gray random visual noise was previewed. The gray lines indicate correct rejection data, and the black lines indicate hit data. 
search was announced aurally, simultaneously with the onset of the nonpreviewed items.

The sequence of trials was random, and the selection of the target item for each trial was pseudorandom. We included a provision that the probability that a previewed item was the target of the search equaled the expected probability. For example, if the subject previewed 1 of the 12 items in the display, the expected probability of that previewed item being the search target was 1 out of 12 . Given that there were 12 trials in the 1-previewed-item condition, we constrained the program so that the previewed item ended up being the target for 1 of these 12 trials. Similarly, a previewed item was the search target in 2 of the 12 trials in the 2 -previewed-items condition.

The subjects hit one key to indicate that the target had appeared in the display and a second key when the target was absent from the display. The RT, which started when the target was announced and ended with the keypress, was recorded for each trial.

\section{Results}

Mean RTs for 26 subjects are presented in Figure 7. The figure shows a fairly linear decrease in RT as the number of previewed items increased from one to three items. Adding more than three items produced no additional benefit. These results suggest that adding information to the previewed display helps an observer in his/her subsequent response as long as there is capacity left in which to represent that information. However, after about three items, the capacity of the representation is exceeded, and adding information to the preview does not result in more information being added to the representation. These data are consistent with the interpretation of Experiments 1 and 2 that the representation created during preview is capacity limited to about three items. ${ }^{3}$

\section{GENERAL DISCUSSION}

Our results suggest that exposure to a scene does not automatically produce an awareness of the identity of all the objects present in that scene (in the sense of an abstract representation that can be interrogated by subsequent cog-

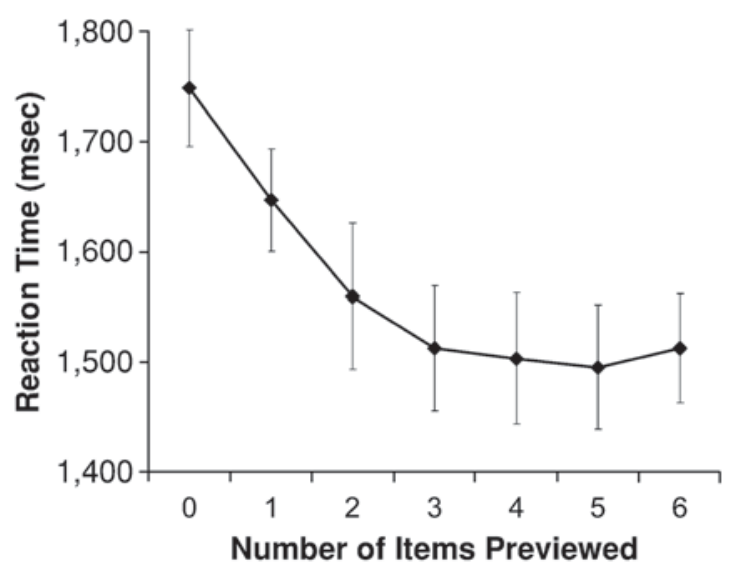

Figure 7. Reaction time for hits as a function of the number of items displayed during preview in Experiment 3. Adding more items to the preview initially reduced reaction time, but the benefit reached a plateau at three items. nitive processes without requiring continued processing of newly arriving visual input). Instead, the identities of only a few items (presumably, those to which an observer is attending) are represented. In the present situation, this set appeared to number about three items; naturally, this number might be somewhat different for different kinds of materials or displays. These findings are wholly consistent with claims based on change blindness that people's visual representations are fairly limited, as compared with the contents of a complex display (Levin \& Simons, 1997; O’Regan et al., 2000; O’Regan et al., 1999; Pashler, 1988; Rensink et al., 1997; Simons \& Levin, 1997). Although our conclusions support common interpretations of change detection studies, the method we used did not require subjects to simultaneously represent two scenes and make comparisons between these representations. Thus, we contend that the present results provide much more clear-cut evidence of the impoverished nature of visual representations ${ }^{4}$ when people view a single scene and suggest that the conclusion very likely applies to the ubiquitous situations outside of the laboratory in which people view a scene with spatially diffuse attention and have the goal of comprehending or understanding the scene as a whole.

\section{Alternative Interpretations}

An alternative interpretation of our results is that subjects could potentially have had access to the identities of more than three items but used only the representations of three items, for strategic reasons. According to this interpretation, observers may not always use all of the information that they have represented; instead, they may adopt different strategies, perhaps depending on the amount of effort required to use memory, as compared with the difficulty of a visual task. In other experimental designs, there is some evidence that subjects will alter their strategies and show more evidence of reliance on memory if a visual task is made impossible (Wolfe et al., 2000) or more difficult (Ballard, Hayhoe, Pook, \& Rao, 1997). For instance, Ballard et al. had subjects copy a model by selecting and arranging different colored blocks. When the model was placed close to the location where the subjects were constructing their copy, the subjects made frequent eye movements back to the model, suggesting very little use of memory. However, when the model was moved $70^{\circ}$ into the periphery, the subjects made fewer eye movements to the model and seemed to rely more heavily on their memory.

Indeed, as we mentioned in the introduction, one of the reasons for embedding the search array in visual noise was to make the visual task more difficult, in order to entice the subjects to use whatever memory representations they had. It is possible, however, that our noise manipulation made the visual task sufficiently hard to entice the subjects to use some (about three items worth), but not all, of their memory representations. Although this account cannot be dismissed, it seems rather unlikely, given that both our strong and our weak noise manipulations resulted in the subjects using the same number of items. If 
the subjects represented more than three items and chose how many of those items to use on the basis of the difficulty of the visual task, one should have expected that the subjects would use more of the memory representation in Experiment 1, which contained more severe noise. It certainly seems most parsimonious to suppose that three is the number of items that they had available. We cannot, however, rule out the possibility that both levels of noise enticed the subjects to access memory but that the subjects were not sufficiently sensitive to the difference between the levels of noise to adopt different numbers of items to access from memory.

A second alternative interpretation of our findings might be that the subjects were capable of encoding all the identities in the display but the 2-sec preview was not long enough for them to do so. Although this is remotely possible, it seems most unlikely that encoding of these materials would be so slow. For instance, Gegenfurtner and Sperling (1993) varied the SOA between the onsets of a cue that indicated which row of a search array to report and a mask. The number of items people were able to encode before the mask appeared suggested a $100 \mathrm{msec}$ per item encoding rate. This estimate predicts that $2 \mathrm{sec}$ of preview should allow the encoding of 20 items.

It is possible that embedding our stimuli in noise significantly slowed this encoding process, allowing only three items to be encoded. However, this seems most unlikely, given that the two different levels of noise used in Experiments 1 and 2 produced the same capacity estimates. If an encoding process that was sensitive to visual noise determined the capacity of the representation, one would predict that the less severe noise manipulation in Experiment 2 should have allowed more items to be represented. It did not.

Instead, it seems likely that the observers often identified many more than three items during the preview or, at least, could have done so but maintained the representation of only three items (cf. Becker \& Pashler, 2002). If so, our data are consistent with Wolfe et al.'s (2000) claim that postattentive visual representations "revert back" to their preattentive state. They are congenial to the view that some items identified during preview are subsequently lost when attention is switched to new objects and are inconsistent with the view that a person attends to different parts of a scene in order to build up a more complex and detailed representation of the scene. Instead, attention may be used in a goal-directed fashion, as a tool to extract a fleeting representation of details about a scene (see Hayhoe, 2000; Hayhoe, Bensinger, \& Ballard, 1998).

This conclusion, however, seems at odds with a theory recently proposed by Hollingworth and colleagues, which claims that visual representations are far more complete (Hollingworth, 2003, 2004; Hollingworth \& Henderson, 2002; Hollingworth et al., 2001). Under Hollingworth et al.'s theory, visual representations are composed of both the limited contents of VSTM and the relatively high capacity contents of VLTM. The theory claims that abstracted representations of the visual contents of the scene are spatially indexed in VLTM; by fixating a particular location in the scene, one can retrieve these abstracted contents. Our data are not consistent with this model, as applied to the kinds of displays presented in these experiments. If observers used the preview time to store letter identities in a spatially indexed VLTM representation and these identities were activated by refixating the item, we would have expected preview of a noisy array to eliminate the effect of noise or change the search efficiency to some efficiency associated with the speed of memory retrieval. We found neither an elimination of the effect of noise nor a change in the search efficiency following preview.

Instead, our data suggest that preview allows only a very limited number of object identities to be represented in such a way that observers can access those identities without having to return to the scene and perform a visual search. We find that only three item identities are immediately available to an observer after previewing a display; this capacity limit of about three items is wholly consistent with VSTM (Luck \& Vogel, 1997; Pashler, 1988; Phillips, 1983) and does not indicate the existence of a larger capacity VLTM system.

We cannot, however, rule out the possibility that item identities are stored in VLTM during preview. It is possible that VLTM representations of the item identities were stored but that their retrieval was either too slow or required too much effort to be used in this task. If so, our findings suggest that the VLTM system may play a limited role in typical scene viewing. The visual noise used in our experiments should have biased people to use an internal representation if they had one. If people fail to use such a representation in this task, it is difficult to believe that they rely on such a representation when viewing typical scenes that are less noisy. Consistent with this view, the methods (Hollingworth, 2003, 2004; Hollingworth \& Henderson, 2002; Hollingworth et al., 2001) used to demonstrate the existence of VLTM were not speeded tasks, and the information the observer needed to complete the task was no longer available in the scene. These tasks may have forced people to use a slower, more effortful system that is rarely used when the scene is present.

It may be important to note that in the present study artificial arrays of characters were used, whereas in the studies taken to support a role for VLTM naturalistic scenes have typically been used. It is possible that observers would show significantly more benefit of preview and more use of VLTM with such scenes-for example, because the greater variation in content might reduce associative interference.

Although we can not rule out the possibility that VLTM might play an important role with different kinds of displays, our data fail to show evidence of a readily available and detailed representation of scenes following preview. Instead, they favor the interpretation that the immediately available contents of visual representations are not complex but represent only a small subset of currently attended items.

The most radical interpretation of the present results and earlier change detection results is that there is absolutely no information available about unattended items. Clearly, 
this interpretation is an overstatement of the results. The results show that people fail to represent unattended items with enough detail to perform tasks that require explicit knowledge about the identities and/or the locations of specific items within a display. The failure to find evidence of this type of representation does not necessarily indicate that there is no representation of unattended stimuli, but only that whatever representation there is about these stimuli is insufficient to perform these tasks.

There are a number of other types of findings that demonstrate that extensive visual analysis can be done in parallel across the entire visual field. For example, in Experiment 2, the observers were able to "clean up" visual noise without the need for focal attention. In addition, many search experiments have shown that discrepant elements often pop out of an array of distractors, without the need for focal attention. This pop-out can occur when the target item differs from distractors on a basic feature, such as size, brightness, or orientation (Treisman \& Gelade, 1980), but it may also occur for features that require more extensive processing. For example, differences in stimulus properties conveyed through shape from shading can lead to pop-out (Enns \& Rensink, 1990), as can differences in perceived size based on size-distance scaling (Ramachandran, Pashler, \& Plummer, 1990). It has also been argued that higher level analysis (e.g., identification of characters) can support parallel visual search (Pashler \& Badgio, 1985; Sperling \& Melchner, 1978; Wolfe, FriedmanHill, Stewart, \& O'Connell, 1992). All of these findings suggest that far more than three items in a display can undergo extensive and abstract analysis at the same time.

\section{Implications for Interpreting Search Data}

When analyzing visual search data, many researchers emphasize the slopes of set size functions without fully considering the $y$-intercept of those functions (see Wolfe, 1998 , for a review). It is not clear, however, that one can disregard this intercept effect and interpret only the search slopes.

In our data, preview did not decrease the slopes of the set size functions. If we had relied exclusively on the slope data to interpret our results, we would have concluded that observers acquire no useful information during preview. However, if we had ignored the slope data and based our interpretations solely on the $y$-intercepts of the search functions, we would have reached dramatically different conclusions. In all of our experiments, we find shorter overall RTs when there has been preview; thus, we would conclude that preview allows a subject to represent information that informs the subsequent search. These two interpretations, based on different aspects of the data, lead to conclusions that appear to be in direct opposition.

However, if we look at both slopes and intercepts concurrently, a more complete and cohesive explanation is potentially available. The intercept effect shows that there is some benefit of preview, suggesting that observers are able to extract the identity of some, but not all, of the items during preview, thus lowering the overall RT. On the other hand, the failure to find any change in slopes with preview suggests that preview does not offer any useful processing for items whose identities are not represented. Furthermore, applying this model to the data from Experiments 1 and 2 allowed us to estimate that only about three item identities are represented. Experiment 3 confirmed this capacity limit, using a different method. It may be that similar explanations could be developed to account for other results that contain seeming conflicts between slope and intercept results (e.g., Wolfe et al., 2000; Woodman, Vogel, \& Luck, 2001).

\section{Conclusions}

Our results provide particularly strong evidence that visual representations acquired while a scene is viewed are sparse and fragile, apparently (in our task, at least) representing the identity of no more than about three items at any instant. These results cannot be attributed to an inability to simultaneously represent two scenes or to an inability to make comparisons between scenes; thus, the results go well beyond so-called change blindness findings in their implications. In addition, we find that examining both search slopes and $y$-intercepts concurrently helps to offer a more complete picture of the processes underlying a present/absent search judgment.

\section{REFERENCES}

Ballard, D. H., Hayhoe, M. M., Pook, P. K., \& Rao, R. P. N. (1997). Deictic codes for the embodiment of cognition. Behavioral \& Brain Sciences, 20, 723-767.

Becker, M. W., \& PAshler, H. (2002). Volatile visual representations: Failing to detect changes in recently processed information. Psychonomic Bulletin \& Review, 9, 744-750.

Becker, M. W., Pashler, H., \& Anstis, S. M. (2000). The role of iconic memory in change-detection tasks. Perception, 29, 273-286.

Dennett, D. C. (1991). Consciousness explained. Boston: Little, Brown.

DennetT, D. C. (2002). How could I be wrong? How wrong could I be? Journal of Consciousness Studies, 9, 13-16.

Egeth, H., \& Dagenbach, D. (1991). Parallel versus serial processing in visual search: Further evidence from subadditive effects of visual quality. Journal of Experimental Psychology: Human Perception \& Performance, 17, 551-560.

EnNs, J. T., \& Rensink, R. A. (1990). Sensitivity to three-dimensional orientation in visual search. Psychological Science, 1, 323-326.

Fernandez-Duque, D., \& Thornton, I. M. (2002). Explicit mechanisms do not account for implicit localization and identification of change: An empirical reply to Mitroff et al. (2002). Journal of Experimental Psychology: Human Perception \& Performance, 29, 846-858.

Gegenfurtner, K. R., \& Sperling, G. (1993). Information transfer in iconic memory experiments. Journal of Experimental Psychology: Human Perception \& Performance, 19, 845-866.

Hayhoe, M. M. (2000). Vision using routines: A functional account of vision. Visual Cognition, 7, 43-64.

Hayhoe, M. M., Bensinger, D. G., \& Ballard, D. H. (1998). Task constraints in visual working memory. Vision Research, 38, 125-137.

HollingWORTH, A. (2003). Failures of retrieval and comparison constrain change detection in natural scenes. Journal of Experimental Psychology: Human Perception \& Performance, 29, 388-403.

HoLLINGWORTH, A. (2004). Constructing visual representations of natural scenes: The roles of short- and long-term visual memory. Journal of Experimental Psychology: Human Perception \& Performance, 30 , 519-537.

Hollingworth, A., \& Henderson, J. M. (2002). Accurate memory for previously attended objects in natural scenes. Journal of Experimental Psychology: Human Perception \& Performance, 28, 113-136. 
Hollingworth, A., Williams, C. C., \& Henderson, J. M. (2001). To see and remember: Visually specific information is retained in memory from previously attended objects in natural scenes. Psychonomic Bulletin \& Review, 8, 761-768.

Johnsen, A. M., \& Briggs, G. E. (1973). On the locus of display load effects in choice reactions. Journal of Experimental Psychology, 99, 266-271.

Levin, D. T., \& Simons, D. J. (1997). Failure to detect changes to attended objects in motion pictures. Psychonomic Bulletin \& Review, 4, 501-506.

LoGAN, G. D. (1978). Attention in character-classification tasks: Evidence for the automaticity of component stages. Journal of Experimental Psychology: General, 107, 32-63.

LuCK, S. J., \& VogeL, E. K. (1997). The capacity of visual working memory for features and conjunctions. Nature, 390, 279-281.

Mewhort, D. J. K., Johns, E. E., \& Coble, S. (1991). Early and late selection in partial report: Evidence from degraded displays. Perception \& Psychophysics, 50, 258-266.

Mitroff, S. R., Simons, D. J., \& Franconeri, S. L. (2002). The siren song of implicit change detection. Journal of Experimental Psychology: Human Perception \& Performance, 28, 798-815.

O'Regan, J. K. (1992). Solving the "real" mysteries of visual perception: The world as an outside memory. Canadian Journal of Psychology, 46, 461-488.

O'Regan, J. K., Deubel, H., Clark, J. J., \& Rensink, R. A. (2000). Picture changes during blinks: Looking without seeing and seeing without looking. Visual Cognition, 7, 191-212.

O'Regan, J. K., \& Nö̈, A. (2001). A sensorimotor theory of vision and visual consciousness. Behavioral \& Brain Sciences, 24, 939-1031.

O'Regan, J. K., Rensink, R. A., \& Clark, J. J. (1999). Change-blindness as a result of "mudsplashes." Nature, 398, 34.

Pashler, H. (1984). Evidence against late selection: Stimulus quality effects in previewed displays. Journal of Experimental Psychology: Human Perception \& Performance, 10, 429-448.

Pashler, H. (1988). Familiarity and visual change detection. Perception \& Psychophysics, 44, 369-378.

Pashler, H., \& Badgio, P. C. (1985). Visual attention and stimulus identification. Journal of Experimental Psychology: Human Perception \& Performance, 11, 105-121.

PhILlips, W. A. (1983). Short-term visual memory. Philosophical Transactions of the Royal Society of London: Series B, 302, 295-309.

Phillips, W. A., \& Singer, W. (1974). Function and interaction of on and off transients in vision: I. Psychophysics. Experimental Brain Research, 19, 493-506.

Ramachandran, V. S., Pashler, H., \& Plummer, D. (1990, May). Visual pop-out and a 3-D vs. 2-D image features. Paper presented to the Association for Research in Vision and Ophthalmology, Sarasota, FL.

Rensink, R. A., O'Regan, J. K., \& Clark, J. J. (1997). To see or not to see: The need for attention to perceive changes in scenes. Psychological Science, 8, 368-373.

Simons, D. J. (2000). Current approaches to change blindness. Visual Cognition, 7, 1-16.

Simons, D. J., Chabris, C. F., Schnur, T., \& Levin, D. T. (2002). Evidence for preserved representations in change blindness. Consciousness \& Cognition, 11, 78-97.

Simons, D. J., \& Levin, D. T. (1997). Change blindness. Trends in Cognitive Sciences, 1, 261-267.

SPERLING, G. (1960). The information available in brief visual presentation. Psychological Monographs, 74 (11, Whole No. 498).

Sperling, G., \& Melchner, M. J. (1978). Visual search, visual attention, and the attention operating characteristic. In J. Requin (Ed.), Attention and performance VII (pp. 675-686). Hillsdale, NJ: Erlbaum.
Stelmach, L. B., Bourassa, C. M., \& Di Lollo, V. (1984). Detection of stimulus change: The hypothetical roles of visual transient responses. Perception \& Psychophysics, 35, 245-255.

STERnBerG, S. (1969). The discovery of processing stages: Extensions of Donders' method. Acta Psychologica, 30, 276-315.

Thornton, I. M., \& Fernandez-Duque, D. (2000). An implicit measure of undetected change. Spatial Vision, 14, 21-44.

Treisman, A. M., \& Gelade, G. (1980). A feature-integration theory of attention. Cognitive Psychology, 12, 97-136.

Watson, D. G., \& HumPhreYs, G. W. (1997). Visual marking: Prioritizing selection for new objects by top-down attentional inhibition of old objects. Psychological Review, 104, 90-122.

Watson, D. G., Humphreys, G. W., \& Olivers, C. N. L. (2003). Visual marking: Using time in visual selection. Trends in Cognitive Sciences, 7, 180-186.

Wolfe, J. M. (1998). Visual search. In H. Pashler (Ed.), Attention (pp. 13-74). Hove, U.K.: Psychology Press.

Wolfe, J. M., Friedman-Hill, S. R., Stewart, M. I., \& O'Connell, K. M. (1992). The role of categorization in visual search for orientation. Journal of Experimental Psychology: Human Perception \& Performance, 18, 34-49.

Wolfe, J. M., Klempen, N., \& Dahlen, K. (2000). Postattentive vision. Journal of Experimental Psychology: Human Perception \& Performance, 26, 693-716.

Woodman, G. F., Vogel, E. K., \& LucK, S. J. (2001). Visual search remains efficient when visual working memory is full. Psychological Science, 12, 219-224.

\section{NOTES}

1. These estimates are based on the theory that search is a selfterminating serial process. Under this theory, observers will, on average, reach the target of the search halfway through the array. Thus, the slope of the set size search function is .5 of the milliseconds per item search rate. For correct rejections, the observer must search all the items in a display, and thus the slope of the search function is a direct measure of the milliseconds per item search rate.

2. The method of displaying part of the search array before exposing the entire search array seems similar to the method used in visual marking experiments (Watson \& Humphreys, 1997; Watson, Humphreys, \& Olivers, 2003). However, there are two critical differences. First, in visual marking experiments, subjects are aware of the search target from the outset of the trial; thus, they can begin searching for the target as soon as the first array appears. Second, the target is rarely one of the items that is displayed early. Thus, the observer's task in a visual marking experiment is to ignore the locations of the items that appear early, and there is no reason to encode and retain those items' individual identities. By contrast, in our experiment, the subject did not know the identity of the search target until after the entire display was presented and, thus, may have benefitted from encoding the identities of the previewed items.

3. Although the average capacity across subjects is about three items, inspecting individual subjects' data shows that there is variability in the individuals' capacity, varying from about one to five items.

4. Finally, it should be noted that our observers did not have to perform articulatory suppression during the task, so it is possible that the information was being recoded into verbal codes, rather than visual codes. Even if the observers were using verbal codes, the basic conclusion that the capacity of representations held in STM is very limited would hold.

(Manuscript received September 13, 2004; revision accepted for publication February 23, 2005.) 